\title{
CÁLCULO EN LA TRANSFERENCIA DE CALOR Y MASA EN EL SECADO DE LA ANCHOVETA
}

\author{
CALCULATING THE HEAT AND MASS TRANSFER IN DRYING \\ ANCHOVETA
}

\author{
Leonardo A. Sheron Ramírez'; Freddy Delgado Cabrera
}

\begin{abstract}
RESUMEN
La presente investigación tiene por finalidad realizar los cálculos de balance de materia y energía en el secado de anchoveta en un secador de bandejas. El propósito fue desarrollar un modelo matemático para el balance de masa y energía en el proceso de secado. Los objetivos fueron: determinar el balance de masa y calor de pescado sin salar; comparar las curvas de pérdida de peso, en forma práctica y teórica; hacer comparativo el consumo efectivo de energía en el secado. El trabajo se desarrolló en los Laboratorios de Tecnología Pesquera, de la Escuela Académica Profesional de Ingeniería Pesquera y Operaciones Unitarias de la Escuela Académica de Ingeniería en Industrias Alimentarias. El experimento de secado se realizó con anchoveta HG congelada adquirido del supermercado PlazaVea, además se calculó el balance de masa y energía en el proceso de secado.
\end{abstract}

Palabras clave: Secado, balance de masa, balance de energía, $H G$.

ABSTRACT

This research is intended to perform the calculations of mass and energy balance in the drying of anchovies in a tray dryer, with The aim of developing a mathematical model for mass and energy balance in the drying process of anchovy, the objectives are:To determine the mass and heat balance of unsalted fish, compare the curves of weight loss, in a practical and theoretical, and make comparative actual energy consumption in drying the study.Was developed in the laboratories of Fisheries Technology, School of Fisheries Engineering Professional Academic Unit Operations and Academic School Food Engineering Drying experiments was developed HG frozen anchovy acquired PlazaVea supermarket, we performed mass and energy balance in the drying process.

Keywords: drying, mass balance, energy balance, HG.

\section{I.- INTRODUCCIÓN}

El proceso de secado consiste en la remoción de un líquido de un material por la aplicación de calor, llevándose a cabo por evaporación y no por procesos mecánicos. Este proceso se logra por la transferencia del líquido a un gas no saturado como aire. El objetivo del proceso de secado es detener o disminuir el crecimiento de microorganismo así como las reacciones químicas. En alimentos se suele hablar de deshidratación [1][5].

Deshidratación y secado no es lo mismo. Según el Departamento de Agricultura de los Estados Unidos define productos deshidratados aquellos que han sido expuestos a la remoción de agua y que poseen más del $2.5 \%$ de agua (base seca). Productos secos son aquellos con no más del $2.5 \%$ de agua (base seca) [1].

La deshidratación o secado de alimentos no sólo afecta el contenido de agua de alimentos, también altera otras propiedades físicas, químicas y microbiológicas, como la actividad enzimático, crecimiento microbiano, dureza, viscosidad, sabor, palatabilidad [6].

\section{Fenómenos físicos y estructurales}

Los cambios geométricos y estructurales en los alimentos durante el secado afecta las propiedades de transferencia de masa y la calidad del producto. Los defectos más comunes en productos deshidratados son: dureza, textura como madera, rehidratación lenta e incompleta, pérdida de jugosidad (típica de alimentos frescos) y cristalización de polisacáridos como almidones. El 
encogimiento es uno de los cambios más comunes de la deshidratación y ocurre durante las primeras etapas del secado [6].

\section{Aplicaciones del proceso de secado}

El secado de alimentod es reducir el volumen y peso de los materiales obteniendo, de esta manera, la disminución del costo en el transporte y almacenamiento. Adicionalmente ayuda a la conservación y estabilidad de productos animales y vegetales para disminuir el crecimiento de hongos y bacterias. En definitiva, el secado permite obtener un producto más estable, especialmente en alimentos $[7]$.

\section{El proceso de secado}

En los procesos de secado, los datos suelen expresarse como la variación que experimenta el peso del producto que se está secando con el tiempo (observar en el Gráfico $\mathrm{N}^{\circ} \mathrm{O} 1$ ). Aunque a veces, los datos de secado pueden expresarse en términos de velocidad de secado [6].

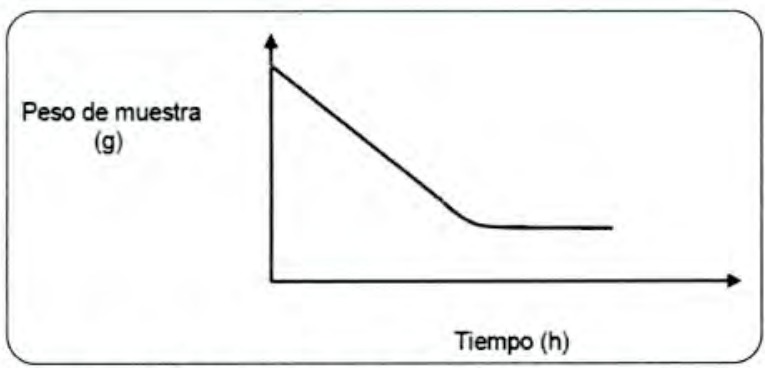

Gráfico $\mathrm{N}^{\circ}$ 01. Cambio de peso durante un proceso de $\operatorname{secado}(6)$

El contenido en humedad del producto se define como la relación entre la cantidad de agua en el alimento y la cantidad de sólidos secos y se expresa como:

$$
\mathrm{Xt}=\frac{(\mathrm{Wt}-\mathrm{Fs})}{\mathrm{Fs}}
$$

En la que Wt es el peso total de material en un tiempo determinado, siendo Fs el peso de los sólidos secos, y Xt es la humedad expresada como peso de agua/peso de sólido seco.

En los procesos de secado una variable muy importante es el denominado contenido de humedad libre X. El contenido de humedad libre se puede evaluar si se considera el contenido de humedad de equilibrio:

$$
\mathrm{X}=\mathrm{Xt}-\mathrm{Xeq}
$$

En la que Xeq es el contenido de humedad cuando se alcanza el equilibrio. Una típica curva de secado se obtiene al representar este contenido de humedad libre $\mathrm{X}$ frente al tiempo de secado $\mathrm{t}$. (observar en el Gráfico $\mathrm{N}^{\circ} \mathrm{O}$ )

La velocidad de secado Rs es proporcional al cambio del contenido de humedad en función del tiempo $(\mathrm{t})$ :

$$
\text { Rs } \alpha \mathrm{dX} / \mathrm{dt}
$$

Considerando la curva presentada en el Gráfico $\mathrm{N}^{\circ} \mathrm{O} 3$, los valores individuales de $\mathrm{dX} / \mathrm{d}$ t en función del tiempo, se pueden obtener a partir de la tangente trazada en la curva de $\mathrm{X}$ frente a $\mathrm{t}$. Sustituyendo la condición de proporcionalidad en la ecuación (3) por Fs/A, la velocidad de secado se puede expresar como:

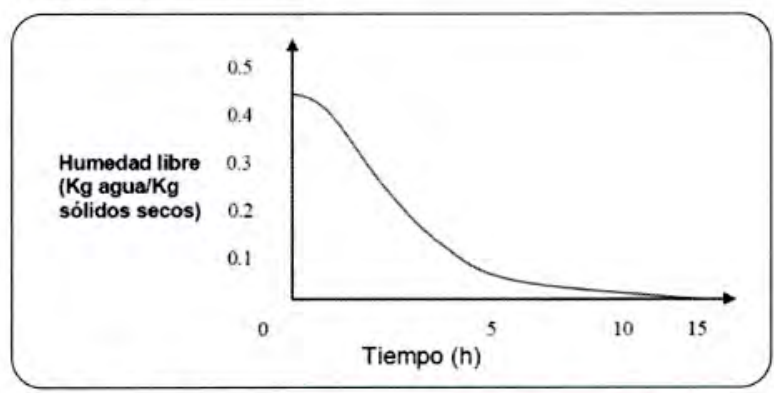

Gráfico $\mathbf{N}^{\circ} \mathbf{0 2}$. Contenido de humedad en función del tiempo de secado(6)

$$
R s=-(F s / A I)(d X / d t)
$$

Donde $\mathrm{R}$ es la velocidad de secado y A1 es el área de la superficie donde tiene lugar el secado. Al representar Rs frente a t se obtiene una curva similar a la que se muestra en el Gráfico $\mathrm{N}^{\circ} \mathrm{O} 2$.

El proceso de secado de un material puede describirse por una serie de etapas en las que la velocidad de secado juega un papel determinante.

En el Gráfico $\mathrm{N}^{\circ} 03$ se muestra una típica curva de velocidad de secado, en la que los puntos A y A' representan el inicio de secado para un material frío y caliente.

El punto $B$ representa la condición de temperatura de equilibrio de la superficie del producto.

El tiempo transcurrido para pasar de A o A' a B suele ser bajo y a menudo se desprecia en los cálculos de tiempo de secado [6]. 


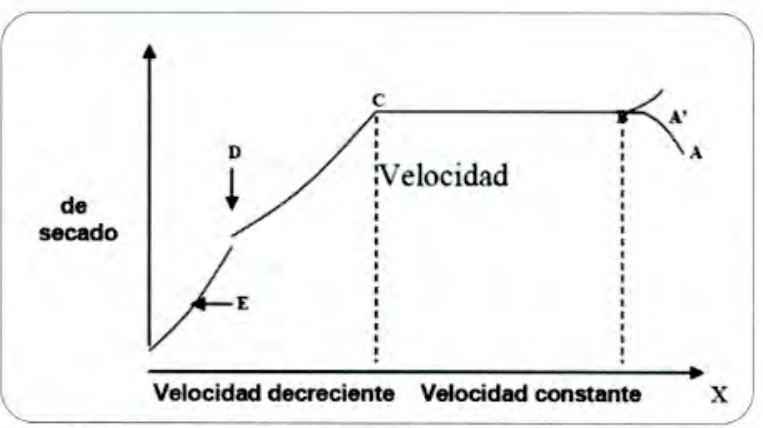

Gráfico $\mathbf{N}^{\circ}$ 03. Curva de velocidad de secado (6)

El tramo de la curva B - C es conocido como periodo de velocidad constante de secado y está asociado a la eliminación del agua no ligada del producto, en el que el agua se comporta como si el sólido no estuviera presente. $\mathrm{Al}$ inicio, la superficie del producto se encuentra muy húmeda, presentando una actividad de agua cercana a la unidad. [6]

En los sólidos porosos, el agua eliminada en la superficie es compensada por el flujo de agua desde el interior del sólido. El período de velocidad constante continúa mientras que el agua evaporada en la superficie puede ser compensada por la que se encuentra en el interior. La temperatura en la superficie se corresponde aproximadamente a la del bulbo húmedo. En general, la velocidad del secado se determina por condiciones externas de temperatura, humedad y velocidad de aire. [6]

El período de velocidad decreciente se da cuando la velocidad del secado ya no se mantiene constante y empieza a disminuir, además, la actividad de agua en la superficie se hace menor que la unidad. En este caso, la velocidad del secado está gobernada por el flujo interno del agua y vapor. $\mathrm{El}$ punto $\mathrm{C}$ del Gráfico $\mathrm{N}^{\circ} \mathrm{O} 3$ representa el inicio del período de velocidad decreciente. En este punto no hay suficiente agua en la superficie para mantener el valor uno de actividad de agua. El período de velocidad decreciente se puede dividir en dos etapas. La primera de ellas se da cuando los puntos húmedos en la superficie disminuyen continuamente hasta que la superficie está seca completamente (punto D), mientras que la segunda etapa del período de velocidad de secado decreciente se inicia en el punto $\mathrm{D}$, cuando la superficie está completamente seca, y el plano de evaporación se traslada al interior del sólido.

El calor requerido para eliminar la humedad es trasferido a través del sólido en la corriente de aire que va hacia la superficie. A veces no existen diferencias remarcables entre el primer y segundo período de velocidad decreciente. [6]
La cantidad de agua eliminada en este período puede ser baja, mientras que el tiempo requerido puede ser elevado, ya que la velocidad de secado es baja. La determinación experimental de la velocidad de secado se basa en un principio simple: medida del cambio del contenido de humedad durante el secado. El material que se desea secar se introduce en una bandeja y es expuesto a una corriente de aire. La bandeja se suspende de una balanza colocada en un armario o conducto por el que fluye aire. El peso del material se va anotando en función del tiempo de secado.

En el Gráfico $\mathrm{N}^{\circ} \mathrm{O} 4$ se muestra un esquema típico utilizado en la determinación de velocidad de secado(6).

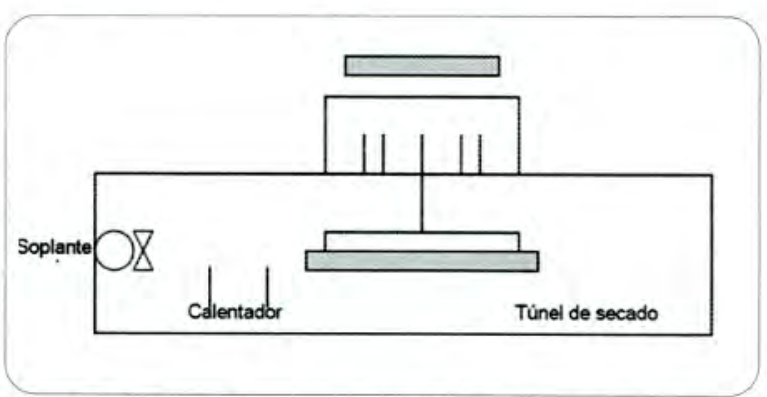

Gráfico $\mathrm{N}^{\circ} \mathbf{0 4}$. Determinación de la velocidad(6)

\section{Determinación de la velocidad de secado}

\section{- Periodo de velocidad constante}

Durante el período de secado de velocidad constante, los fenómenos de transporte que tienen lugar son la transferencia de materia de vapor de agua hacia el medio ambiente, desde la superficie del producto a través de una película de aire que rodea el material y la transferencia de calor a través del sólido.

Mientras dura el proceso de secado la superficie del material permanece saturada de agua, ya que la velocidad del movimiento de agua, desde el interior del sólido, es suficiente para compensar el agua evaporada en la superficie.

Si se supone que sólo existe transferencia de calor hacia la superficie del sólido por convección desde el aire caliente y transferencia de materia desde la superficie hacia el aire caliente (observar en el Gráfico $\mathrm{N}^{\circ} \mathrm{05}$ ) los balances de materia y calor se pueden expresar como:

$$
\begin{aligned}
& \mathrm{Na}=\mathrm{Ky}\left(\mathrm{Yw}_{\mathrm{w}}-\mathrm{Y}\right) \\
& \mathrm{Q}=\mathrm{h} \mathrm{Al}(\mathrm{Tbs}-\mathrm{Tbh})
\end{aligned}
$$




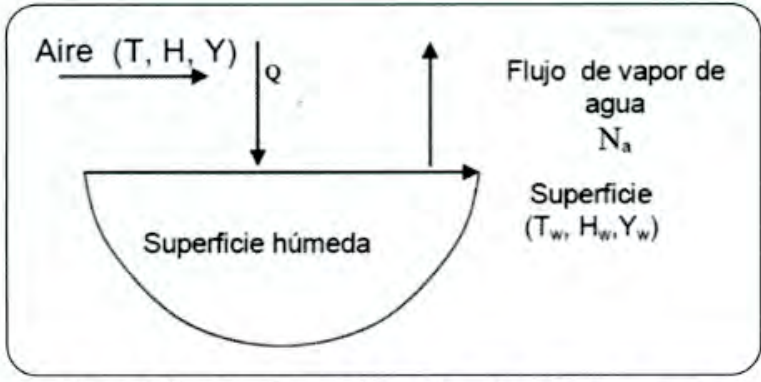

Gráfico $\mathbf{N}^{\circ}$ 05. Transferencia de calor y materia durante el secado(6)

$\mathrm{h}=$ Coeficiente de transferencia de calor $\left(\mathrm{W} / \mathrm{m} 2{ }^{\circ} \mathrm{K}\right)$

A1 = Área de secado $(\mathrm{m} 2)$

Tbh $=$ Temperatura de bulbo húmedo $\left({ }^{\circ} \mathrm{C}\right)$

Tbs $=$ Temperatura de bulbo seco $\left({ }^{\circ} \mathrm{C}\right)$

$\mathrm{Na}=$ Flujo de vapor de agua $(\mathrm{mol} \mathrm{Kg} / \mathrm{sm} 2)$

$\mathrm{Yw}=$ Humedad del aire en la superficie del sólido (Kg agua/Kg aire seco)

$\mathrm{Y}=$ Humedad del aire (Kg agua/ Kg aire seco)

Ky $=$ Coeficiente de transferencia de masa (adimensional)

El Proyecto es importante porque la complejidad de la transferencia de masa y calor en el secado de pescado, debe ser de fácil manejo, por ello pretenderemos realizar los cálculos teóricos y prácticos, de esta manera, tratar de modelar una ecuación matemática, para el secado en un secador de bandejas.

En la teoría de secado de alimentos nos entrega la energía utilizada en el proceso de secado y no nos indica qué cantidad de calor se pierde o se va con el aire de secado.

El método de secado de pescado nos permitió controlar la temperatura en la cámara de secado, tratando de conservar las propiedades nutricionales tanto de proteínas y omega 3.

De esta manera, controlar la temperatura y calor en el secado del pescado, hasta obtener un producto listo para preparar los alimentos.

El trabajo que realizamos es sin la adición de sal en el nuestro producto que va ser evaluado en la determinación de balance de masa y energía.

Con lo manifestado nos hemos planteado la siguiente pregunta:

${ }_{¿}$ Con el uso del secador de bandejas, se puede determinar el balance materia y energía en el secado de anchoveta?

\section{Hipótesis}

Calculo de balance de materia y energía en el secado de anchoveta en un secador de bandejas.

El objetivo general: Desarrollar un modelo matemático para el balance de masa y energía en el proceso de secado de anchoveta.

Los objetivos específicos que plantemos fueron:

- Determinar el balance de masa y calor de pescado sin salar.

- Comparar las curvas de pérdida de peso, en forma práctica y teórica.

- Hacer comparativo del consumo efectivo de energía en el secado.

\section{II.- MATERIALES Y MÉTODOS}

La presente investigación se desarrolló en el Laboratorio de Tecnología Pesquera de la Escuela Académica Profesional de Ingeniería Pesquera de la Universidad Nacional Jorge Basadre Grohmann de Tacna y Laboratorio de Operaciones Unitarias de la Escuela de Ingeniería en Industrias Alimentarias.

Para los experimentos utilizamos como materia prima $\mathrm{HG}$ de anchoveta congelada que fueron adquiridos en el Supermecado Plaza Vea. Se usó 100, 150, 200, 250, 300, 350, 400 y 500 g por tanda, para los experimentos de cálculos de masa y energía en el proceso de secado.

El siguiente diseño experimental se utilizó para la determinación de Balance de masa y energía.

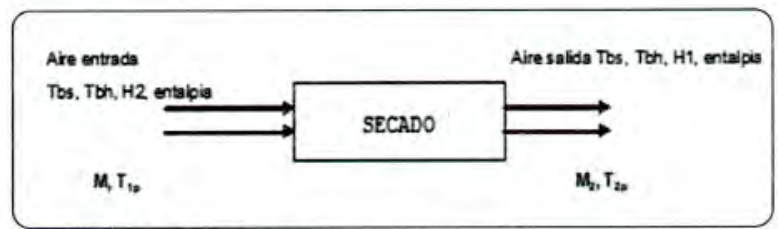

Gráfico $\mathrm{N}^{\circ}$ 06. Diseño experimental 1

Donde:

M Flujo de masa de pescado, $\mathrm{kg} / \mathrm{h}$

$M_{1}$ Flujo de masa a la salida del secador, $\mathrm{kg} / \mathrm{h}$

$\mathrm{M}_{2}$ Flujo de masa de aire $\mathrm{y}$ agua a la salida del secador, $\mathrm{kg} / \mathrm{h}$

Tbs y Tbh temperatura bulbo seco y bulbo húmedo

$\mathrm{T}_{1 \mathrm{p}}$ Temperatura del pescado, ${ }^{\circ} \mathrm{C}$

$\mathrm{T}_{2 p}$ Temperatura del pescado secado, ${ }^{\circ} \mathrm{C}$

$\mathrm{H}_{1}$ Humedad del aire

$\mathrm{H}_{2}$ Humedad del aire a la salida del secador 


\section{III.- RESULTADOS}

Tabla $\mathbf{N}^{\circ} 01$. Pérdida de peso en función al tiempo.

\begin{tabular}{|c|c|c|c|c|c|c|c|c|c|c|}
\hline \multicolumn{2}{|c|}{ Tiempo } & \multicolumn{9}{|c|}{ Experimentos } \\
\hline \multirow{2}{*}{ Min } & \multirow{2}{*}{ h } & E1 & E2 & E3 & E4 & E5 & E6 & E7 & E8 & E9 \\
\hline & & \multicolumn{9}{|c|}{ Pérdida de peso (g) } \\
\hline 0 & 0 & 500,0 & 450,0 & 400,0 & 350,0 & 300,0 & 253,0 & 200,0 & 150,0 & 100,0 \\
\hline 35 & 0,58 & 498,02 & 448,2 & 398,4 & 348,6 & 298,8 & 252,0 & 199,2 & 149,4 & 99,6 \\
\hline 41 & 0,68 & 492,09 & 442,9 & 393,7 & 344,5 & 295,3 & 249,0 & 196,83 & 147,6 & 98,4 \\
\hline 46 & 0,77 & 490,12 & 441,1 & 392,1 & 343,1 & 294,1 & 248,0 & 196,0 & 147,0 & 98,0 \\
\hline 50 & 0,83 & 488,1 & 439,3 & 390,5 & 341,7 & 292,9 & 247,0 & 195,2 & 146,4 & 97,6 \\
\hline 55 & 0,92 & 486,2 & 437,5 & 388,9 & 340,3 & 291,7 & 246,0 & 194,5 & 145,8 & 97,2 \\
\hline 60 & 1,00 & 480,2 & 432,2 & 384,2 & 336,2 & 288,1 & 243,0 & 192,1 & 144,1 & 96,0 \\
\hline 65 & 1,08 & 471,3 & 424,2 & 377,1 & 329,9 & 282,8 & 238,5 & 188,5 & 141,4 & 94,3 \\
\hline 95 & 1,58 & 453,6 & 408,2 & 362,8 & 317,5 & 272,1 & 229,5 & 181,4 & 136,1 & 90,7 \\
\hline 110 & 1,83 & 449,6 & 404,6 & 359,7 & 314,7 & 269,8 & 227,5 & 179,8 & 134,9 & 89,9 \\
\hline 125 & 2,08 & 443,7 & 399,3 & 354,9 & 310,6 & 266,2 & 224,5 & 177,5 & 133,1 & 88,7 \\
\hline 130 & 2,17 & 442,7 & 398,4 & 354,2 & 309,9 & 265,6 & 224,0 & 177,1 & 132,8 & 88,5 \\
\hline 135 & 2,25 & 440,7 & 396,6 & 352,6 & 308,5 & 264,4 & 223,0 & 176,3 & 132,2 & 88,1 \\
\hline 140 & 2,33 & 439,7 & 395,8 & 351,8 & 307,8 & 263,8 & 222,5 & 175,9 & 131,9 & 87,9 \\
\hline 145 & 2,42 & 435,0 & 391,5 & 348,0 & 304,5 & 261,0 & 220,1 & 174,0 & 130,5 & 87,0 \\
\hline 150 & 2,5 & 432,6 & 389,3 & 346,1 & 302,8 & 259,5 & 218,9 & 173,0 & 129,8 & 86,5 \\
\hline 180 & 3 & 418,3 & 376,5 & 334,7 & 292,8 & 251,0 & 211,7 & 167,3 & 125,5 & 83,7 \\
\hline 210 & 3,5 & 404,5 & 364,1 & 323,6 & 283,2 & 242,7 & 204,7 & 161,8 & 121,4 & 80,9 \\
\hline 240 & 4 & 391,2 & 352,1 & 313,0 & 273,8 & 234,7 & 198,0 & 156,5 & 117,4 & 78,2 \\
\hline 300 & 5 & 365,9 & 329,3 & 292,7 & 256,1 & 219,5 & 185,1 & 146,3 & 109,8 & 73,2 \\
\hline 360 & 6 & 342,1 & 307,9 & 273,7 & 239,5 & 205,3 & 173,1 & 136,9 & 102,6 & 68,4 \\
\hline 420 & 7 & 320,0 & 288,0 & 256,0 & 224,0 & 192,0 & 161,9 & 128,0 & 96,0 & 64,0 \\
\hline 540 & 9 & 279,8 & 251,9 & 223,9 & 195,9 & 167,9 & 141,6 & 111,9 & 84,0 & 56,0 \\
\hline 660 & 11 & 244,7 & 220,3 & 195,8 & 171,3 & 146,8 & 123,8 & 97,9 & 73,4 & 48,9 \\
\hline 720 & 12 & 228,9 & 206,0 & 183,1 & 160,2 & 137,3 & 115,8 & 91,6 & 68,7 & 45,8 \\
\hline 780 & 13 & 214,1 & 192,6 & 171,2 & 149,8 & 128,4 & 108,3 & 85,6 & 64,2 & 42,8 \\
\hline 840 & 14 & 200,2 & 180,2 & 160,1 & 140,1 & 120,1 & 101,3 & 80,1 & 60,1 & 40,0 \\
\hline 900 & 15 & 187,2 & 168,5 & 149,8 & 131,0 & 112,3 & 94,7 & 74,9 & 56,2 & 37,4 \\
\hline 960 & 16 & 175,1 & 157,6 & 140,1 & 122,6 & 105,0 & 88,6 & 70,0 & 52,5 & 35,0 \\
\hline 1002 & 16,7 & 167,1 & 150,4 & 133,6 & 116,9 & 100,2 & 84,5 & 66,8 & 50,1 & 33,4 \\
\hline 1005 & 16,75 & 166,5 & 149,8 & 133,2 & 116,5 & 99,9 & 84,2 & 66,6 & 49,9 & 33,3 \\
\hline 1004,4 & 16,74 & 166,6 & 149,9 & 133,3 & 116,6 & 100,0 & 84,3 & 66,6 & 50,0 & 33,3 \\
\hline 1003,5 & 16,725 & 166,8 & 150,1 & 133,4 & 116,7 & 100,1 & 84,4 & 66,7 & 50,0 & 33,4 \\
\hline
\end{tabular}

Fuente: Elaboración propia 


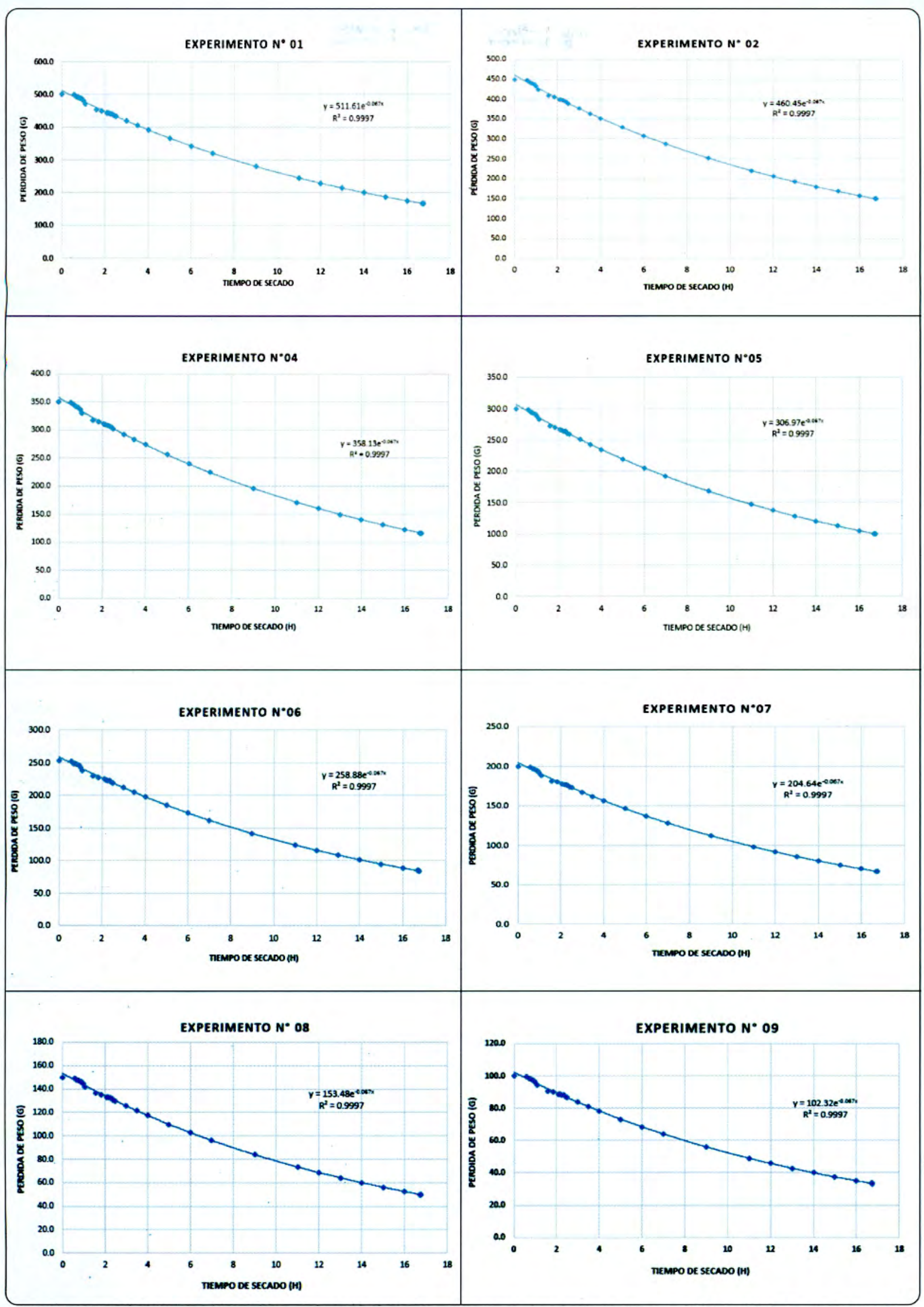

Gráfico $\mathbf{N}^{\circ}$ 07. Comparación de las experiencias realizadas en el secado de anchoveta HG, pérdida en función al tiempò.

Fuente: Elaboración propia 


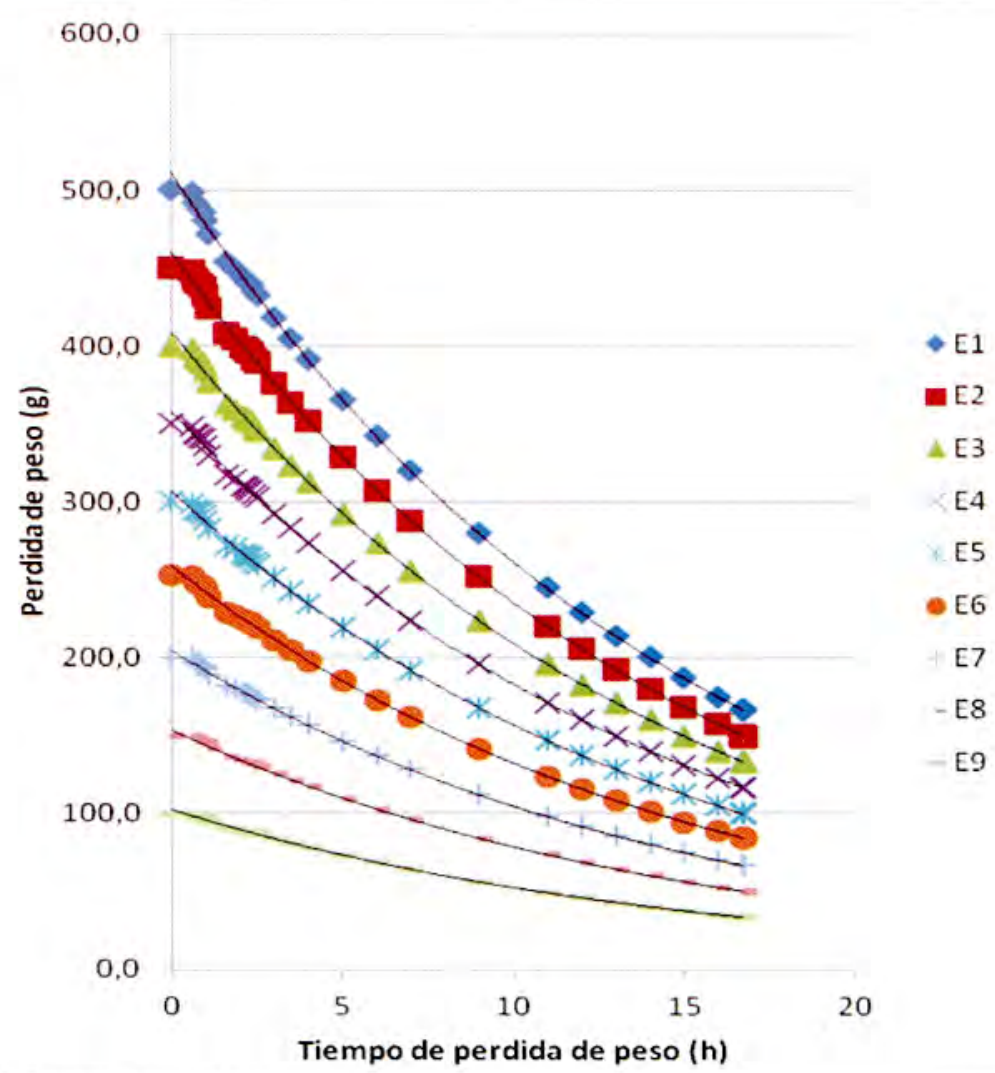

Gráfico $\mathbf{N}^{\circ} \mathbf{0 8}$. Curvas de secado de la anchoveta HG

Fuente: Elaboración propia

Una pérdida de peso de acuerdo a las condiciones de secado (Gráfico $\mathrm{N}^{\circ} \mathrm{O8}$ ).

$\mathrm{Al}$ ingreso del secado temperatura de bulbo seco, $40^{\circ} \mathrm{C}$ y bulbo húmedo, $25^{\circ} \mathrm{C}$, la humedad relativa del aire en el día de los experimentos fue $68 \%$, velocidad del aire de secado fue $1 \mathrm{~m} / \mathrm{s}$. La humedad relativa de acuerdo al diagrama psicrométrico (ver Anexo F) a la salida del calentador del secador es $30 \%$.
La humedad a la entrada del secador de acuerdo a las tablas psicrométrico (ver Anexo F) es $0,016 \mathrm{~g}$ de agua/g de aire seco. A la salida del secador, temperatura de bulbo $\operatorname{seco}, 40^{\circ} \mathrm{C}$ y temperatura de bulbo húmedo $30^{\circ} \mathrm{C}$; la humedad relativa fue $82 \%$ y con una humedad de $0.023 \mathrm{~g}$ de agua/g de aire seco. Los experimentos se realizaron de acuerdo a la Tabla $\mathrm{N}^{\circ} \mathrm{O}$ en el cual se observa el tiempo de secado y las pérdidas de peso.

Tabla $\mathbf{N}^{\circ}$ o2. Balance de masa en las experiencias realizadas en el secado de anchoveta HG.

\begin{tabular}{|l|c|c|c|c|c|c|c|c|c|}
\hline \multirow{2}{*}{ Peso (g) } & \multicolumn{8}{|c|}{ Experiencias } \\
\cline { 2 - 11 } & E1 & E2 & E3 & E4 & E5 & E6 & E7 & E8 & E9 \\
\hline Peso ingreso & 500,0 & 450,0 & 400,0 & 350,0 & 300,0 & 253,0 & 200,0 & 150,0 & 100,0 \\
\hline Agua extraída & 333,2 & 299,9 & 266,6 & 233,3 & 199,9 & 168,6 & 133,3 & 100,0 & 66,6 \\
\hline Peso salida & & & & & & & & & \\
\hline Teórico & 166,7 & 150,0 & 133,3 & 116,7 & 100,0 & 84,3 & 66,7 & 50,0 & 33,3 \\
\hline Práctico & 166,8 & 150,1 & 133,4 & 116,7 & 100,1 & 84,4 & 66,7 & 50,0 & 33,4 \\
\hline Diferencia (\%) & 0,07 & 0,07 & 0,07 & 0,07 & 0,07 & 0,07 & 0,07 & 0,07 & 0,07 \\
\hline
\end{tabular}

Fuente: Elaboración propia 
La humedad del $\mathrm{Hg}$ de anchoveta fue determinada en el Laboratorio de Tecnología Pesquera (producto congelado), $70 \%$.

Como se puede apreciar, el peso de salida con el balance de masa realizado en forma teórica no ha variado significativamente, se observa una constante de $0,07 \%$ de variación.

Se tiene la humedad del aire de entrada y salida los cuales son: $0,016 \mathrm{~g}$ de agua/g de aire seco $\mathrm{y}$ $0,023 \mathrm{~g}$ de agua/g de aire seco, esto nos ayuda a determinar la cantidad de aire necesario, que se requirieron para el secado en las 9 experiencias.

Tabla $\mathrm{N}^{\circ}$ 03. Aire utilizado en el secado de la anchoveta

\begin{tabular}{|c|c|c|c|c|c|c|c|c|c|}
\hline \multirow{2}{*}{ Peso } & \multicolumn{7}{|c|}{ Experiencias } \\
\cline { 2 - 10 } & E1 & E2 & E3 & E4 & E5 & E6 & E7 & E8 & E9 \\
\hline $\begin{array}{c}\text { Aire utilizado } \\
\text { (kg) }\end{array}$ & 47,6 & 42,84 & 38,0 & 33,32 & 28,6 & 24,01 & 19,0 & 14,28 & 9,52 \\
\hline $\begin{array}{c}\text { Aire utilizado } \\
\text { (Kg) }\end{array}$ & 47,6 & 42,8 & 38,1 & 33,3 & 28,6 & 24,1 & 19,0 & 14,3 & 9,5 \\
\hline $\begin{array}{c}\text { Aire utilizado } \\
\text { (\%) }\end{array}$ & 0,29 & 0,26 & 0,23 & 0,20 & 0,17 & 0,15 & 0,12 & 0,09 & 0,06 \\
\hline
\end{tabular}

Fuente: Elaboración propia

En la Tabla $\mathrm{N}^{\circ} 03$, la densidad del aire de acuerdo al diagrama psicrométrico, $1,092 \mathrm{~kg} / \mathrm{m}^{3}$; flujo volumétrico $0,25 \mathrm{~m}^{3} / \mathrm{s}$; velocidad del aire $1 \mathrm{~m} / \mathrm{s}$; dimensiones del ventilador $0,5 \mathrm{mx0}, 05 \mathrm{~m}$ (área, $\left.0,25 \mathrm{~m}^{2}\right) ; 900 \mathrm{~m}^{3} / \mathrm{h}$; flujo másico, $982,8 \mathrm{~kg} / \mathrm{h}$; aire de entrada, $16437,3 \mathrm{~kg}$.

De acuerdo al consumo de aire (entrada) y usado efectivamente, se tiene un consumo de efectivo de 0,06 a $0,23 \%$, para el secado.

Tabla $\mathrm{N}^{\circ}$ 04. Entalpía del aire a la entrada y salida del secador

\begin{tabular}{|l|c|}
\hline \multicolumn{1}{|c|}{ Condiciones } & Entalpia \\
\hline Entrada & $54 \mathrm{~kJ} / \mathrm{kg}$ de aire seco \\
\hline Salida & $70 \mathrm{~kJ} / \mathrm{kg}$ de aire seco \\
\hline
\end{tabular}

Fuente: Elaboración propia

En la Tabla $\mathrm{N}^{\circ} 04$ observamos, respecto al balance de energía y del diagrama psicrométrico, tenemos la entalpía a temperatura de bulbo seco y temperatura de bulbo húmedo, a la entrada 54 $\mathrm{kJ} / \mathrm{kg}$ y a la salida $70 \mathrm{~kJ} / \mathrm{kg}$ de aire seco. Con el flujo volumétrico, $0,25 \mathrm{~m}^{3} / \mathrm{s}$, el volumen $/ \mathrm{kg}$ de aire es $0,91 \mathrm{~m}^{3} / \mathrm{kg}$. Con la variación de entalpía a $24 \mathrm{~kJ} / \mathrm{kg}$ de aire seco y el volumen másico, tenemos: $17,5824176 \mathrm{~kJ} / \mathrm{m}^{3}$ que se transfieren al calentamiento del aire. Con el flujo volumétrico tenemos el flujo calórico, $15824,17582 \mathrm{~kJ} / \mathrm{h}$; con el tiempo de proceso $(16,75 \mathrm{~h})$, tenemos el consumo de energía $265054,945 \mathrm{~J}(265,0549 \mathrm{~kJ})$; $73,49973626 \mathrm{kWh}$.
Tabla $\mathrm{N}^{\circ} 05$. Energía utilizada en las experiencias

\begin{tabular}{|c|c|c|c|c|c|c|c|c|c|}
\hline \multirow{2}{*}{ Peso } & \multicolumn{7}{|c|}{ Experiencias } \\
\cline { 2 - 10 } & $E 1$ & $E 2$ & $E 3$ & $E 4$ & E5 & E6 & E7 & E8 & E9 \\
\hline $\begin{array}{c}\text { Peso ingreso } \\
\text { (kg) }\end{array}$ & 0,5 & 0,45 & 0,40 & 0,35 & 0,30 & 0,25 & 0,20 & 0,15 & 0,10 \\
\hline $\begin{array}{c}\text { Calor } \\
\text { necesario } \mathrm{kJ}\end{array}$ & 0,072 & 0,0644 & 0,057 & 0,050 & 0,043 & 0,036 & 0,0286 & 0,0215 & 0,014 \\
\hline $\begin{array}{c}\text { Porcentaje } \\
\text { utilizado }\end{array}$ & 0,027 & 0,024 & 0,022 & 0,019 & 0,016 & 0,014 & 0,011 & 0,008 & 0,01 \\
\hline
\end{tabular}

Fuente: Elaboración propia

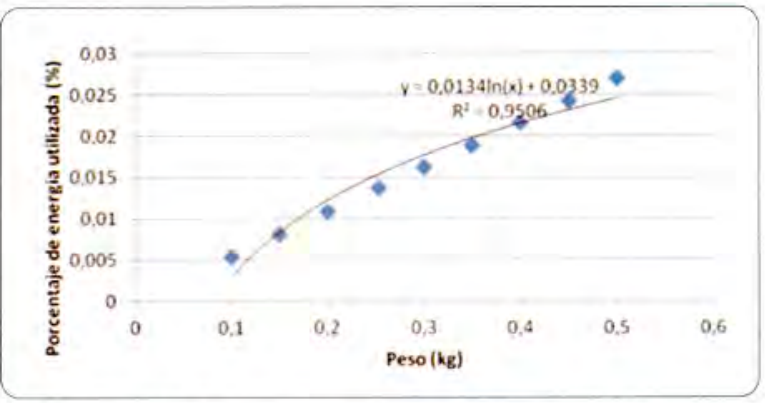

Gráfico $\mathrm{N}^{\circ}$ 09. Peso de anchoveta $\mathrm{HG}$ versus porcentaje utilizado de energía.

Fuente: Elaboración propia

En la Tabla $\mathrm{N}^{\circ} 05$ y el Gráfico $\mathrm{N}^{\circ} 09$, observamos que el porcentaje de uso de energía es logarítmica, a medida que se incrementa el peso de la materia prima, aumenta la energía usada en el secado.

\section{IV.-DISCUSIÓN}

Barbosa et al [8][9] nos indica qué tipo de curva es. En nuestro trabajo hemos encontrado curvas de pérdida de peso en función al tiempo de secado. Tenemos curvas exponenciales.

Con respecto a trabajos similares no nos fue posible encontrar referencias.

Con respecto a la energía utilizada, vemos que, a medida que se aumenta la carga de materia prima a secar, el aire cumple su característica secante.

De las experiencias realizadas, el secador de bandejas nos entrega datos que se pueden procesar.

El consumo de energía efectivo sucede de acuerdo a la cantidad de materia prima, aumenta de acuerdo al incremento de peso. En el presente trabajo, va de $0,027 \%$ a $0,01 \%$. La función es logarítmica.

\section{V.- CONCLUSIONES}

- Según las experiencias de secado de anchoveta HG, se encontró que el modelo es exponencial, con un coeficiente de correlación muy aceptable. 
- Los modelos determinados nos indican que a medida que se incrementa la materia prima, el poder secante del aire aumenta.

- El peso de producto seco práctico es cercano al teórico, pues la variación es $0,07 \%$, el cual es aceptable.

- El aprovechamiento de la energía calórica del aire se incrementa a medida que se aumenta el peso de la materia prima a secar, se presenta una función logarítmica.

\section{VI.- RECOMENDACIONES}

Se recomienda continuar con otros trabajos tecnológicos, para obtener productos, listos para consumir, secados condimentados en especial la anchoveta que es un recurso con una cantidad apreciable de proteínas y omegas.

\section{REFERENCIAS BIBLIOGRÁFICAS}

[1] Treybal, Robert F. Las operaciones de transferencia de masa. México: Person Prentice Hall, 1990. ISBN.

[2] Himmelblau, D.M. Principios Básicos y cálculos en Ingeniería Químic a. México: Person Prentice Hall, 2002. ISBN.

[s] Singh, R.P. y Heldman, D.R. Introduction to Food Engineering. s.l.: Elsevier Inc., 2009. ISBN.

[4. Toledo, R.T. Fundamentals of Food Process Engineering. New York-USA: Springer

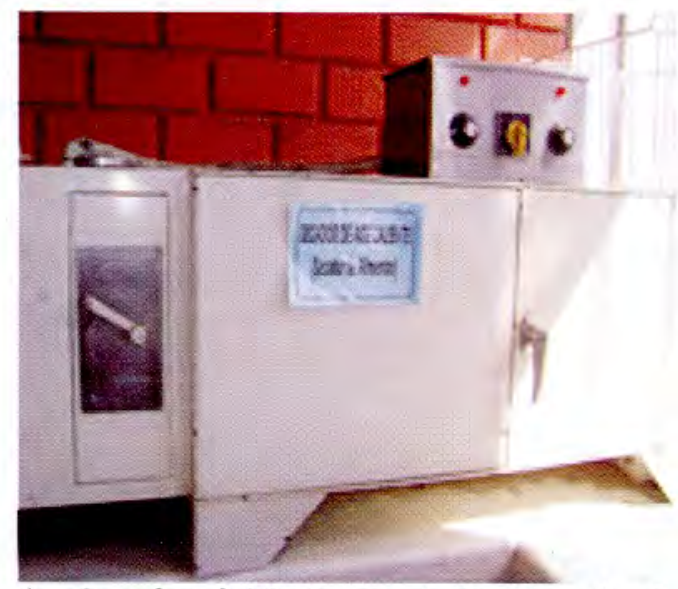

A.- Secador del Laboratorio de Tecnología Pesquera.
Science Busines Media, LLC, 2007. ISBN.

[5] Smith, P.G. Introduction to Food Process Engineering. London: Springer New York Dordrecht Heidelberg, 2011 . ISBN.

[6]Barbosa-Canovas, G.V. Métodos Experimentales de la Ingeniería Alimentaria. Zarogoza-España: ACRIBIA S.A., 2007. ISBN.

[7] Geankoplis, Christie John. Procesos de Transporte y principios de Procesos de Separación. México: Compañía Editorial Continental, 2006. ISBN.

[8] Barbosa-Canovas, G.V., Vega-Mercado, H y Ibarz-Ribas, A. Deshidratación de alimentos. Zaragoza-España: ACRIBIA, 2000. ISBN.

[9]Barbosa-Cánovas, G.V. Métodos experimentales de la Ingeniería Alimentaria. Zaragoza-España: ACRIBIA S.A., 2007. ISBN.

\section{Correspondencia:}

Leonardo A. Sheron Ramírez

Ciudad Universitaria fundo "Los Granados"

Av. Miraflores s/n Tacna - Perú

Freddy Delgado Cabrera

Ciudad Universitaria fundo "Los Granados"

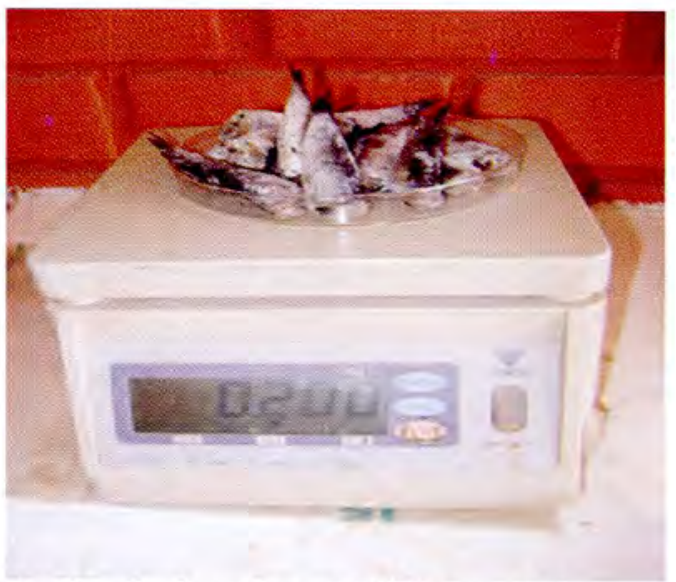

B.- Pesado de anchoveta HG congelada. 

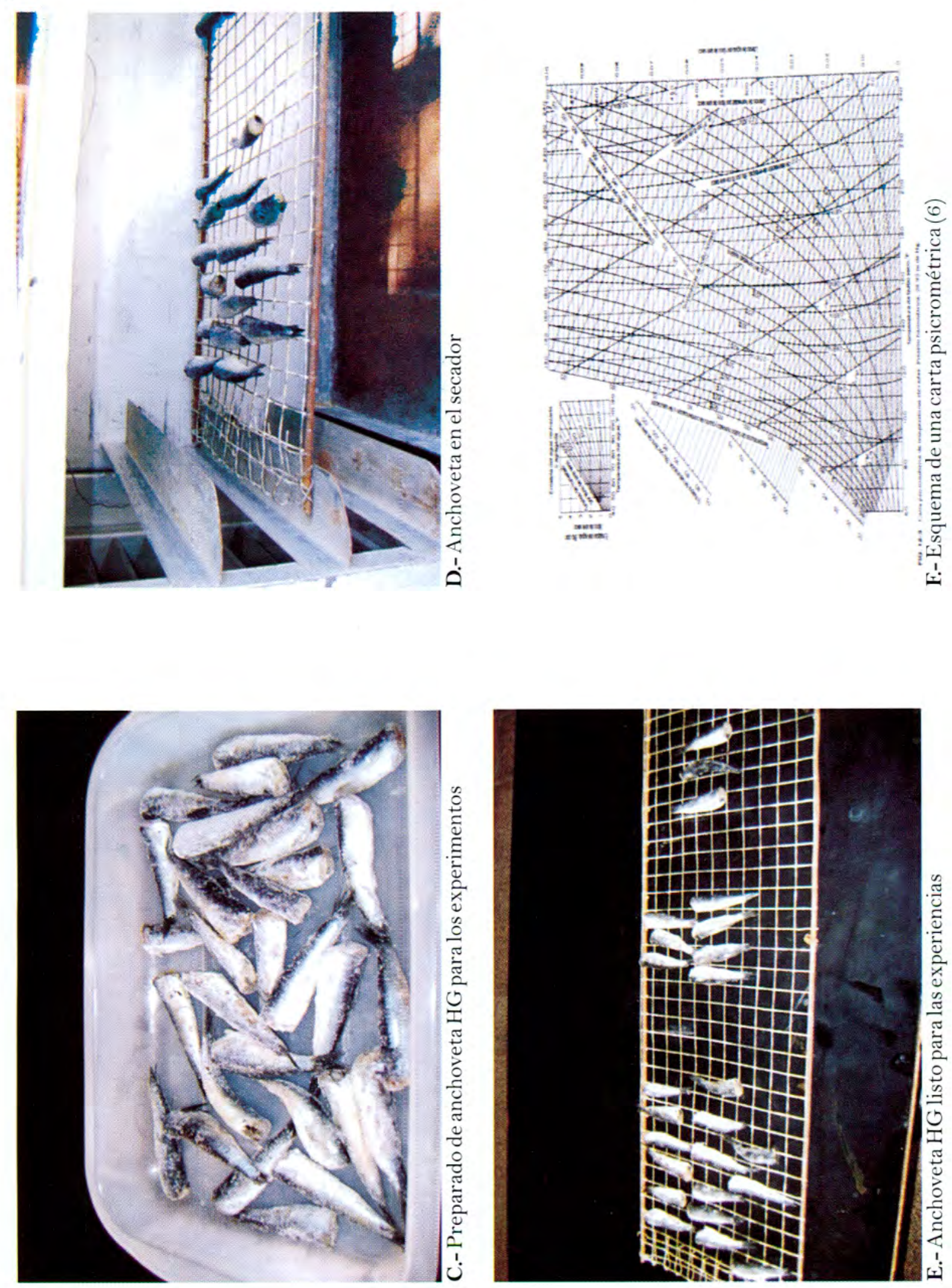